\title{
The Ala-Tragus Line as a Guide for Orientation of the Occlusal Plane in Complete Dentures
}

\author{
${ }^{1}$ Carole Abi-Ghosn, ${ }^{2}$ Carla Zogheib, ${ }^{3}$ Ronald Younes, ${ }^{4}$ Joseph E Makzoumé
}

\section{ABSTRACT}

Aim: The use of the ala-tragus line (ATL) to orient the occlusal plane $(\mathrm{OP})$ is controversial because there is a lack of agreement on the exact points of reference for this line. This study determined the relationship between the natural OP and ATL which was established by using the inferior border of the ala of the nose and (1) the superior border of the tragus (ATL1), (2) the tip (ATL2), and (3) the inferior border of the tragus (ATL3).

Materials and methods: Lateral cephalometric radiographs were taken of each subject by a standard method and tracings were obtained on acetate paper to show the OP and the three ala-tragus lines. The relationship between the OP and each of ATL was measured for each subject. Mean and standard deviation values were then calculated for the relationship. Statistical analysis was performed using repeated measure analysis of variance followed by Bonferroni pairwise comparisons and Student's t-test $(\alpha=0.05)$.

Results: Significant differences were noted between the three mean angles $(p=0.046)$. The mean angle $(3.275$ degrees \pm $2.54)$ formed by OP and ATL2 was significantly the smallest $(p<0.05)$.

Conclusion: The ala-tragus line, extending from the inferior border of the ala of the nose to the tip of the tragus of the ear presented the closest relationship to the natural occlusal plane.

Keywords: Edentulous, ala of the nose, tragus of ear, occlusal plane, complete denture.

The ala-tragus line as a guide for orientation of the occlusal plane in complete dentures.

How to cite this article: Abi-Ghosn C, Zogheib C, Younes R, Makzoumé JE. The Ala-Tragus Line as a Guide for Orientation of the Occlusal Plane in Complete Dentures. J Contemp Dent Pract 2014;15(1):108-111.

${ }^{1}$ Private Practice and Senior Lecturer, ${ }^{2,3}$ Private Practice and Assistant Professor, ${ }^{4}$ Private Practice, Associate Professor and Chairman

${ }^{1}$ Department of Removable Prosthodontics, School of Dentistry Saint-Joseph University, Beirut, Lebanon

${ }^{2}$ Department of Endodontics, Saint-Joseph University, Beirut Lebanon

${ }^{3}$ Department of Surgery, Saint-Joseph University, Beirut Lebanon

${ }^{4}$ Department of Removable Prosthodontics, School of Dentistry Saint-Joseph University, Beirut, Lebanon

Corresponding Author: Carole Abi-Ghosn Yared, Private Practice and Senior Lecturer, Department of Removable Prosthodontics, School of Dentistry, Saint-Joseph University, Beirut, Lebanon, e-mail: caroleabighosn@hotmail.com

\section{Source of support: Nil}

Conflict of interest: None

\section{INTRODUCTION}

The occlusal plane (OP) is defined as 'the average plane established by the incisal and occlusal surfaces of the teeth'. Several principles have been postulated for determining the OP; however, no single method seems to be fully accepted. ${ }^{2-9}$ Although criticized, Camper's line or ala-tragus line (ATL) is a widely used guide for OP orientation. ${ }^{10,11}$ Many studies have analyzed orientation of OP in complete the denture construction, ${ }^{12-15}$ and the $\mathrm{OP}$ in relation to craniofacial reference planes. ${ }^{16-21}$

During denture fabrication, the OP is generally established in the patient's mouth in reference to anatomic or physiologic landmarks. One common method to establish OP in clinical practice involves dividing it into 3 segments, one anterior and 2 posterior. The anterior segment of $\mathrm{OP}$ is usually determined according to esthetic and phonetic criteria. It is then rendered parallel to a line connecting pupils of the eyes. Posterior segments are made parallel to the ATL which is defined as 'a line running from inferior border of ala of the nose to some defined point on ear tragus, usually considered to be the tip of tragus'. ${ }^{1}$ However, it is not unusual for clinicians to utilize the superior or the inferior border of the tragus. ${ }^{22,23}$ Obviously, there is confusion over posterior reference points of the ATL. The purpose of this study was to determine relationship between natural OP and ATL which was established by using inferior border of the ala and (1) superior border of the tragus (ATL1), (2) tip (ATL2) and (3) inferior border of the tragus (ATL3).

\section{MATERIALS AND METHODS}

Lateral cephalometric radiographs were taken for each participant using a standard method and tracings were obtained on acetate paper to show OP and the three ala-tragus lines (Fig. 1). The relationship between the OP and each of ATL was measured for each subject. Mean and standard deviation values were then calculated for the relationship.

After institutional review board approval, 20 dental students were included in this study. Subjects ranged from 21 to 24 years with a mean age of 22.55 . Criteria for selection 


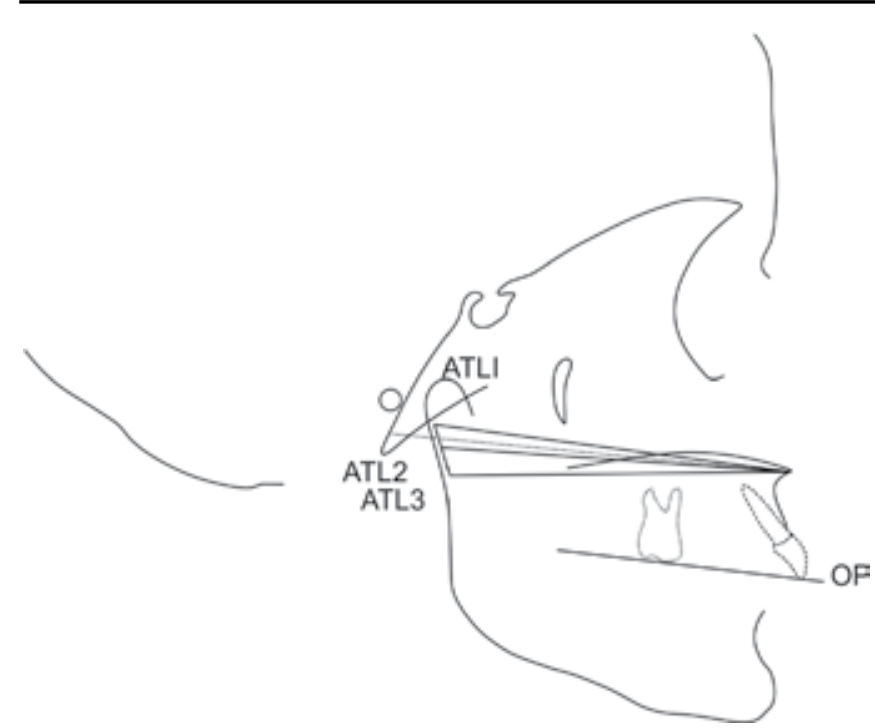

Fig. 1: Tracing of a cephalogram showing position of natural occlusal plane and the three ala-tragus lines: ATL1: Ala-tragus line extending from inferior border of ala of nose to the superior border of tragus of ear; ATL2: Ala-tragus line extending from inferior border of ala of nose to center of tragus of ear; ATL3: Ala-tragus line extending from inferior border of ala of nose to inferior border of tragus of ear; OP: Natural occlusal plane

of these students were the presence of 28 to 32 natural teeth in an acceptable arch alignment, with Angle's class I relationship and no history of orthodontic treatment.

Standard lateral cephalometric radiographs of each subject were made with a cephalostat (Panoramic Corporation. PC1000, Forwayne-Indiana USA, range capability of 70 to 90 $\mathrm{KV}$, at $10 \mathrm{~mA}$ for 0.4 to 5.0 seconds). The cone film distance used was 5 feet. Exposure was 80 to $90 \mathrm{KV}$ at $10 \mathrm{~mA}$ for 1.5 to 2 seconds in accordance with the subject's physical stature. All radiographs were taken with the mandible in slight occlusion. The landmarks used in this study were as follows:

1. ATL1: Line extending from inferior margin of ala of nose to superior border of tragus of ear.

2. ATL2: Line extending from inferior margin of ala of nose to tip of tragus of ear.

3. ATL3: Line extending from inferior margin of ala of nose to inferior border of tragus of ear.

4. Natural occlusal plane: Line extending from mesioincisal angle of maxillary central incisor to mesiopalatal cusp of first maxillary molar.

Identification of exact points of reference on radiographs was made possible by using small radiopaque ball-shaped pellets (1 mm diameter). Pellets were attached (1) with adhesive tape to the inferior margin of the ala of the nose and to the three posterior reference points for the ATL and (2) with sticky wax to the tip of the mesiopalatal cusp of the maxillary first molar and to the mesioincisal angle of maxillary central incisor.

Tracings were made on acetate paper from all radiographs. The deviation between the natural OP and each of the ATL was measured two times by each of the authors. The angles formed in the anterior region were given a positive value.

The statistical analysis was performed by a software program (SPSS for Windows, Version 16.0, Chicago, IL). Repeated measure analysis of variance followed by Bonferroni pairwise comparisons were conducted to explore differences between mean angles. To find out if ATL1, or ATL2 or ATL3 is parallel to OP, Student's t-test was used comparing each mean angle with the value 0 degree. Significance level was set at 0.05 .

\section{RESULTS}

Table 1 lists the relationship between the natural OP and ATL1, ATL2 and ATL3 for all 20 subjects. Statistical analysis of results is shown in Table 2. Significant difference was found between the three mean angles $(p=0.046)$. The mean angle formed by the OP and ATL2 (3.275 degrees \pm 2.54 ) was significantly the smallest $(p<0.05)$, followed by the mean angle formed by OP and ATL3 (4.675 degrees \pm 2.90 ). The mean angle formed by OP and ATL1 (6.125 degrees \pm 3.62$)$ was significantly the highest.

No parallelism was found between the natural OP and ATL1 ( $<<0.001)$, or ATL2 $(\mathrm{p}<0.001)$, or ATL3 $(\mathrm{p}<0.001)$.

Table 1: Relationship between the natural occlusal plane and the ala-tragus line

\begin{tabular}{|c|c|c|c|}
\hline Subject & $\begin{array}{l}\text { Angle (degrees) } \\
\text { between OP } \\
\text { and ATL1 }\end{array}$ & $\begin{array}{l}\text { Angle } \\
\text { (degrees) } \\
\text { between OP } \\
\text { and ATL2 }\end{array}$ & $\begin{array}{l}\text { Angle (degrees) } \\
\text { between } \\
\text { OP and } \\
\text { ATL3 }\end{array}$ \\
\hline 1 & 4 & 2 & 7.5 \\
\hline 2 & 12 & 8.5 & 4.5 \\
\hline 3 & 5.5 & 2 & 0.5 \\
\hline 4 & 7 & 2 & 3.5 \\
\hline 5 & 8 & 4 & 2 \\
\hline 6 & 6 & 2 & 3.5 \\
\hline 7 & 5 & 1.5 & 7 \\
\hline 8 & 6.5 & 2.5 & 1.5 \\
\hline 9 & 5.5 & 1 & 3 \\
\hline 10 & 1.5 & 4 & 9 \\
\hline 11 & 9 & 2 & 5 \\
\hline 12 & 10 & 5.5 & 3 \\
\hline 13 & 10.5 & 7 & 2.5 \\
\hline 14 & 5 & 0.5 & 6 \\
\hline 15 & 2.5 & 2 & 6.5 \\
\hline 16 & 4 & 0.5 & 3 \\
\hline 17 & 2 & 2 & 6.5 \\
\hline 18 & 2 & 1.5 & 6 \\
\hline 19 & 2 & 7 & 12 \\
\hline 20 & 14.5 & 8 & 1 \\
\hline
\end{tabular}




\begin{tabular}{lccl}
\hline \multicolumn{4}{c}{ Table 2: Statistical analysis of results } \\
\hline Type of composite & Mean & $\begin{array}{l}\text { Standard } \\
\text { deviation }\end{array}$ & $\begin{array}{l}\text { Bonferroni } \\
\text { pairwise } \\
\text { comparisons* }\end{array}$ \\
\hline $\begin{array}{l}\text { Angle (degrees) } \\
\text { between OP and }\end{array}$ & 6.125 & 3.62 & $\mathrm{a}$ \\
ATL1 & & & \\
$\begin{array}{l}\text { Angle (degrees) } \\
\text { between OP and }\end{array}$ & 3.275 & 2.54 & $\mathrm{~b}$ \\
ATL2 & & & \\
$\begin{array}{l}\text { Angle (degrees) } \\
\text { between OP and } \\
\text { ATL3 }\end{array}$ & 4.675 & 2.90 & $\mathrm{a}, \mathrm{b}$ \\
\hline
\end{tabular}

*Same letters indicate no significant difference

\section{DISCUSSION}

Should the OP correspond to a sole, fixed and precise position in each individual, no clinician would ever hope find this position when fabricating complete dentures. Several authors believe that prosthetic OP must be oriented as closely as possible to the OP that existed with natural dentition. ${ }^{8,9}$ By so doing the tongue and cheeks will be more effective during deglutition and mastication and speech and esthetics will be improved. In a limited study conducted on 8 subjects to test masticatory efficiency when the orientation of the OP was varied, Carey found that function was not appreciably affected and that a certain amount of leeway is permissible when orienting the OP. ${ }^{12}$ Levin stated that many patients have the adaptive capacity for a slight change in the plane of occlusion (1 to $2 \mathrm{~mm}$ at most) that sometimes can improve stability for a poor ridge. ${ }^{8}$

Results of this study have shown no parallelism between the natural OP and any of the defined ATL. Abrahams and Carey found the angle between the natural OP and ATL1 to be 9.66 degrees $\pm 4.29 .{ }^{22}$ While Karkazis and Polyzois reported 5.25 degrees \pm 3.35 for the same angle, this study has shown 6.12 degrees $\pm 3.62 .^{14}$

In earlier reports, values of the angle between the natural OP and ATL2 were found to be 2.75 degrees \pm 3.64 and 2.88 degrees $\pm 3.54 .{ }^{14,23}$ The mean angle obtained in this study was 3.27 degrees \pm 2.54 . Such relatively narrow limits show a close relationship between the two planes.

In a similar research to this one, Karkazis and co-workers found the deviation between the natural OP and ATL 3 to be 0.5 degrees \pm 3.55 , while this study indicated 4.67 degrees $\pm 2.90 .{ }^{23}$ These authors reported that ATL3 presented the closest relationship to the natural OP, whereas this study has shown ATL2. The discrepancy could be due to the different determination of the posterior landmarks of the ATL. Karkazis and associates identified those points directly on radiographs by means of the superior (ATL1), center (ATL2) and inferior (ATL3) borders of the metal rods of the ear fixation pins. Obviously, the location of the OP in complete denture construction is a controversial issue..$^{20,21}$

From all these results, it can be inferred that the reliability of ATL as a sole reference to orient the OP is questionable. Clinical reality demands concrete, practical and accurate guide for clinicians to help them determine a lost $\mathrm{OP}$ in the edentulous. Unfortunately, such a method does not exist. ATL can be used safely as a reference for OP determination during fabrication of complete dentures only if combined with other methods for determining the OP. Retro molar pad, lateral borders of the tongue, bisected space between residual alveolar ridges... may also help to assess the orientation of the prosthetic OP. Karkazis and Polyzois and Van Niekerk and co-workers stated that with increasing experience there is less and less reliance on specific guides; there is rather a perception of a 'correctness' in the work. ${ }^{13,14}$ Positioning of the OP depends on mature clinical judgment of the individual clinician and must ultimately satisfy esthetics, function, comfort and denture stability.

\section{CONCLUSION}

The findings of this study indicated that the ala-tragus line, extending from the inferior border of the ala of the nose to the tip of the tragus of the ear presented the closest relationship to the natural occlusal plane.

\section{ACKNOWLEDGMENTS}

The authors wish to thank Dr Fouad Nakhlé and Dr Nada Osta for their statistical assistance

\section{REFERENCES}

1. Glossary of prosthodontic terms. 8th ed. J Prosthet Dent 2005; 94:10-92.

2. Merkeley HJ. The labial and buccal accessory muscles of mastication. J Prosthet Dent 1954;4:327-334.

3. Yasaki M. The height of the occlusion rim and the interocclusal distance. J Prosthet Dent 1961;11:26-31.

4. Nagle RJ, Sears YH. Denture prosthetics. 2nd ed. St Louis: Mosby; 1962. p.134.

5. Ismail YH, Bowman JF. Position of the occlusal plane in natural and artificial teeth. J Prosthet Dent 1968;20:407-411.

6. Lundquist DO, Luther WW. Occlusal plane determination. J Prosthet Dent 1970;23:489-498.

7. Beresin VE, Schiesser FJ. The neutral zone in complete and partial dentures. 2nd ed. St Louis: Mosby; 1978. p.78-79.

8. Levin B. A reevaluation of Hanau's Laws of Articulation and the Hanau Quint. J Prosthet Dent 1978;39:254-258.

9. Spratley MH. A simplified technique for determining the occlusal plane in full denture construction. J Oral Rehabil 1980;7: 31-33.

10. Monteith BD. A cephalometrically programmed adjustable plane: A new concept in occlusal plane orientation for complete denture patients. J Prosthet Dent 1985;54:388-394. 
11. D'Souza NL, Bhargava K. A cephalometric study comparing the occlusal plane in dentulous and edentulous subjects in relation to the maxillomandibular space. J Prosthet Dent 1996;75:177-182.

12. Carey PD. Occlusal plane orientation and masticatory performance of complete dentures. J Prosthet Dent 1978;39: 368-371.

13. Van Niekerk FW, Miller VJ, Chem C, Bibby RE. The ala-tragus line in complete denture prosthodontics. J Prosthet Dent 1985; 53:67-69.

14. Karkazis HC, Polyzois GL. A study of the occlusal plane orientation in complete denture construction. J Oral Rehabil 1987; 14:399-404.

15. Celebic A, Valentic-Peruzovic M, Kraljevic K, Brkic H. A study of the occlusal plane orientation by intraoral method (retromolar pad). J Oral Rehabil 1995;22:233-236.

16. L'Estrange PR, Vig PS. A comparative study of the occlusal plane in dentulous and edentulous subjects. J Prosthet Dent 1975;33:495-503.

17. Ow RKK, Djeng SK, Ho CK. Orientation of the plane of occlusion. J Prosthet Dent 1990;64:31-36.
18. Karkazis HC, Polyzois GL. Cephalometrically predicted occlusal plane: implications in removable prosthodontics. J Prosthet Dent 1991;65:258-264.

19. Nissan J, Barnea E, Zeltzer C, Cardash HS. Relationship between occlusal plane determinants and craniofacial structures. J Oral Rehabil 2003;30:587-591.

20. Hindocha AD, Vartak VN, Bhandari AJ, Dudani M. A Cephalometric Study to Determine the Plane of Occlusion in Completely Edentulous Patients: Part I. J Indian Prosthodont Soc. 2010 December;10(4):203-207.

21. Rosati R, Rossetti A, De Menezes M, Ferrario V, Sforza C. The occlusal plane in the facial context: interoperator repeatability of a new three-dimensional method. J Oral Sci 2012;4: 34-37.

22. Abrahams R, Carey PD. The use of the ala-tragus line for occlusal plane determination in complete dentures. J Dentistry 1979; 7:339-341.

23. Karkazis HC, Polyzois GL, Zissis AJ. Relationship between alatragus line and natural occlusal plane. Implications in denture prosthodontics. Quintessence Int 1986;17:253-255. 